\title{
MedChatBot: An UMLS based Chatbot for Medical Students
}

\author{
Hameedullah Kazi \\ Isra University \\ Hyderabad \\ Pakistan
}

\author{
B.S. Chowdhry \\ Mehran University of \\ Engineering \& Technology \\ Jamshoro, Pakistan
}

\author{
Zeesha Memon \\ Isra University \\ Hyderabad \\ Pakistan
}

\begin{abstract}
The use of natural dialog has great significance in the design of interactive tutoring systems. The nature of student queries can be confined to a small set of templates based on the task domain. This paper describes the development of a chatbot for medical students, that is based on the open source AIML based Chatterbean. We deploy the widely available Unified Medical Language System (UMLS) as the domain knowledge source for generating responses to queries. The AIML based chatbot is customized to convert natural language queries into relevant SQL queries. The SQL queries are run against the knowledge base and results returned to the user in natural dialog. Student survey was carried out to identify various queries posed by students. The chatbot was designed to address common template queries. Knowledge inference techniques were applied to generate responses for queries for which knowledge was not explicitly encoded. Query responses were rated by three experts on a 1-5 point likert scale, who agreed among themselves with Pearson Correlation Coefficient of 0.54 and $p<0.05$. The overall average rating assigned by experts was 3.4.
\end{abstract}

\section{General Terms}

Chatbot, medical tutoring systems, UMLS, AIML, natural dialog, ontology

\section{Keywords}

Chatbot, medical tutoring systems, UMLS, AIML, natural dialog, ontology

\section{INTRODUCTION}

A chatbot is a computer program that interacts with human users using natural language. Chatbots facilitate easier learning of the domain concepts and their relationships, and are particularly relevant for students in the case of an educational domain. User queries are typically questions that are run against the knowledge base to retrieve corresponding answers.

Beginning with developments such as ELIZA [1], the use of chatbots has been witnessed in a diverse set of applications. Knill et al. [2] report the usage of a chatbot for helping students in solving problems related to calculus. Jia [3] employs a chatbot for teaching foreign languages. Kerly et al. [4] describe how a chatbot can be deployed in the context of an intelligent tutoring system for $\mathrm{C}$ language, which supports refinement of the student model through natural language conversation. Webber [5] developed a chatbot that simulates a patient in giving natural language responses to medical students inquiring the patient. Shawar \& Atwell [6] surveyed various chatbot systems and have argued that while they serve as very useful tools in different domains such as education, information retrieval and entertainment, they may not be able to replace humans in the foreseeable future.
The use of Artificial Intelligence Markup Language (AIML) has made great strides in the development of chatbots, beginning with chatbots such as Alicebot [7]. AIML supports natural language dialog by matching an input query to a text pattern which is associated to a template response. The response in the template is returned for the input query that matches the associated pattern.

The design of a chatbot typically requires extensive knowledge acquisition, which is eventually stored into the system to support the user queries. Rules of question answer response are often hard coded or stored into the knowledge base, such that the system is only able to retrieve answers to the questions that are explicitly encoded into it and it is unable to support knowledge extraction.

In this paper we describe the design of an easy to build chatbot for medical students. This chatbot can easily be incorporated in a tutoring system to support natural dialog for effective pedagogy. We describe how an existing knowledge source such as the UMLS can be used to generate query responses through knowledge extraction and by using the open source chatterbean chatbot [8].

\section{AIML BASED CHATBOT}

Chatterbean is an open source java based AIML interpreter. We customize chatterbean to support the relevant queries through AIML. The format of AIML is as follows:

$<$ category>

$$
\begin{aligned}
& <\text { pattern }>\text { what is a ball? }</ \text { pattern }> \\
& <\text { template }>\text { It is a toy }</ \text { template }>
\end{aligned}
$$

$</$ category $>$

In the example above, the query "What is a ball?" results in the response "It is a toy." Symbolic reduction involves the process of simplifying complex grammatical forms into simpler ones. It is used to simplify one form of a query into another form. For example:

<category>

$<$ pattern $>$ What is a ball?</pattern $>$

$<$ template $>$ It is a toy $</$ template $>$

$</$ category $>$

$<$ category>

<pattern>Do you know what a ball is?</pattern>

$<$ template $><$ srai $>$ What is a ball $?</$ srai $></$ template $>$ $</$ category $>$

In the example above, the query, "Do you know what a ball is?" is reduced to the query, "What is a ball?" 


\section{UNIFIED MEDICAL LANGUAGE SYSTEM (UMLS)}

The UMLS [9] is a widely available medical knowledge source and is essentially a collation of various medical ontologies and terminologies (MeSH, SNOMED-CT, Gene Ontology, etc). UMLS contains about two million medical concepts covering various medical domains and 135 semantic types, where each medical concept is assigned at least one semantic type. For example, the concept heart attack has semantic type disease or syndrome, whereas the concept swelling has semantic type finding.

The UMLS consists of the following components. The metathesaurus, which is a collection of concepts and terms and their relationships; the semantic network, which is a set of categories and relationships to classify and relate the entries in the Metathesaurus; the specialist lexicon, which is a database of lexicographic information for use in natural language processing.

Our system makes use of the UMLS tables: mrconso, mrdef, mrsty, and mrcoc. The table mrconso contains the full list of medical concepts in the UMLS Metathesaurus; the table mrdef contains the textual definition of the medical concepts; the table mrsty contains the semantic types of the concepts; the table mrcoc contains the co-occurrence of medical concepts found in medline citations.

\section{SYSTEM PROTOTYPE}

The UMLS [9] is a widely available medical knowledge source and is essentially a collation of various medical ontologies and terminologies (MeSH, SNOMED-CT, Gene Ontology, etc). UMLS contains about two million medical concepts covering various medical domains and 135 semantic types, where each medical concept is assigned at least one semantic type. For example, the concept heart attack has semantic type disease or syndrome, whereas the concept swelling has semantic type finding.

The system prototype is designed by customizing the existing open source chatterbean chatbot. Figure 1 shows a view of the system prototype.

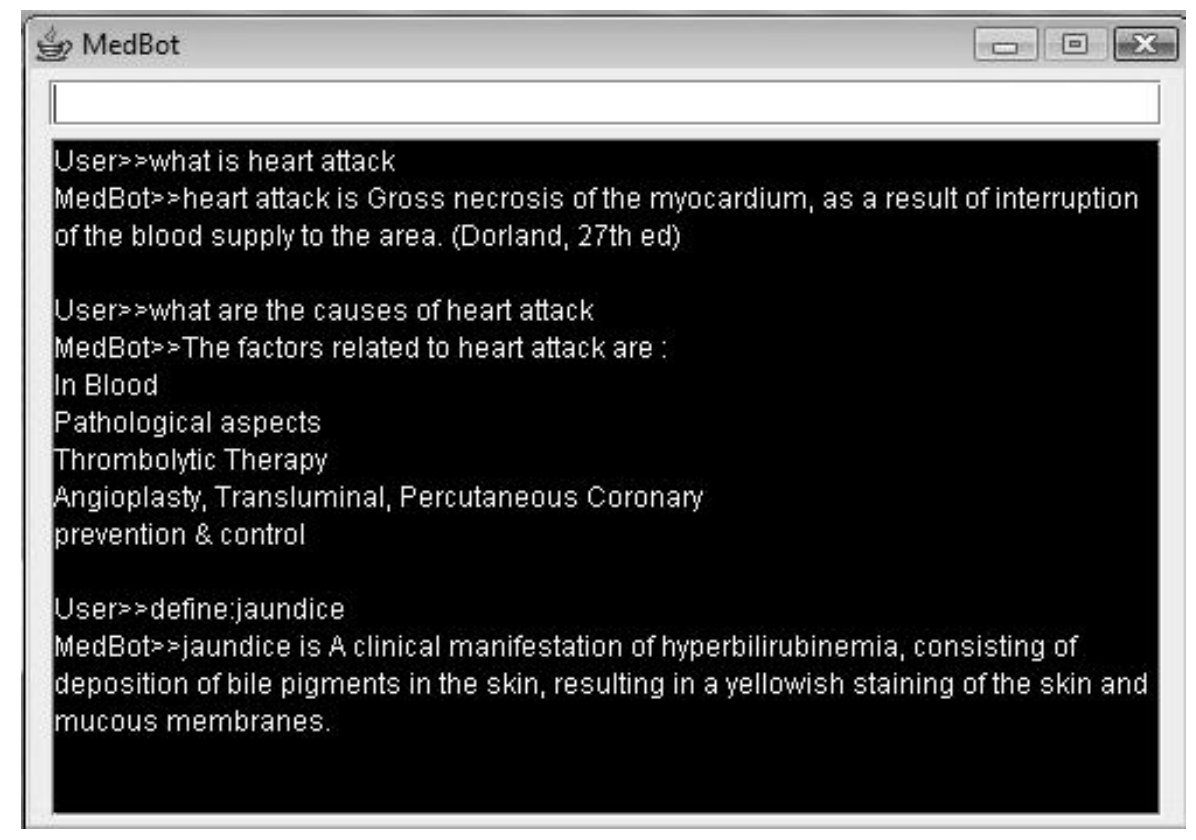

Figure 1: Prototype Interface of MedChatBot

The architecture of MedChatBot consists of three major components: the front end, the AIML parser and the database. The system works as follows.

The user enters a question in natural language. AIML parser converts question into an AIML pattern, identifies the correct pattern using pattern matching technique and then searches and returns wildcard symbol (keyword) to the front-end. The front-end formulates a SQL query using keyword and disposes that query to the database. The database processes query and returns results to the front-end. In the end, the front-end combines the information extracted from database with the template (bot's automated answer) returned by the AIML parser.

\section{METHODS}

A user survey was conducted to identify the possible questions users would pose to a chatbot. The survey was presented to medical students at Isra University. A total of 97 question samples were collected and then those questions were divided into categories depending on the type of question. The resultant categories were ranked according to the number of questions in each category. The majority of questions were based on what is query, comprising $47 \%$ of the posed questions. The remaining categories comprised less than $7 \%$ each of the total questions.

The classification shows that mostly users ask "what is $<$ concept $>$ ?" questions when they are interacting with a 
chatbot. The what is query could be posed in many different ways:

Do you know about <concept>?

What do you know about <concept>?

Define <concept $>$

Can $\mathrm{u}$ tell me something about $<$ concept $>$ ?

What about <concept>?

<concept>?

Thus we decided to implement this category of questions in the chatbot along with three other categories. The other three categories, what is the type of, what are the causes of and what are the symptoms of are also implemented because they could be answered by exploiting the knowledge structure in UMLS.

Thus, the AIML templates are encoded to support four types of queries: what is, what is the type of, what are the causes of, and what are the symptoms of. The chatbot was designed to support the most common queries described above, by encoding the relevant AIML templates. Responses to what is query are provided by extracting the textual definition of the relevant concept found in UMLS. For example, as shown in Figure 1, the query what is heart attack leads to the response: heart attack is gross necrosis of the myocardium ... Responses to what is the type of query are provided by retrieving the semantic type of the relevant concept in UMLS. Responses to what are the causes of and what are the symptoms of are generated through knowledge extraction by employing a method described by Liu \& Chu [10].

If two concepts A and B are found to have co-occurrence as evident from UMLS table mrcoc, we check their semantic types and see if there is a causal relationship between their semantic types. See Figure 2. For example, if the query is based on what are the causes of concept B, we check which concepts have co-occurrence with concept $\mathrm{B}$. If concept A is found to have co-occurrence with concept $B$, we check the semantic type of both concepts. If semantic type A has a causes relationship with semantic type $\mathrm{B}$, we infer that concept A causes concept B.

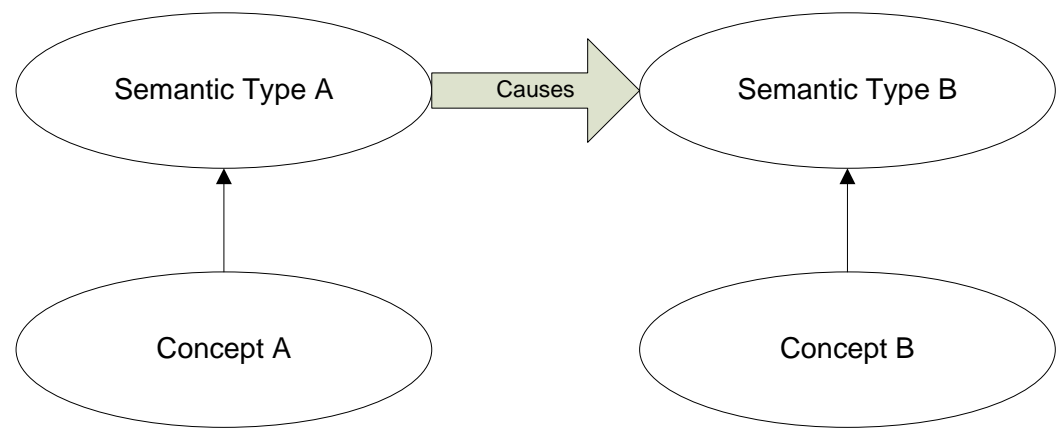

Figure 2: Relationships between Semantic Types of Concepts A and B

\section{RESULTS}

Five randomly selected medical students were asked to provide a list of 30 queries each based on the categories described above. From the 150 queries, 13 consisted of what are the causes of, 28 of what are the symptoms of, 96 of what is, and 13 of what is the type of queries. The queries along with their system generated responses were then given to three faculty members in the Faculty of Medicine \& Allied Medical Sciences at Isra University. These medical experts rated the responses on a scale of $1-5$, where 1 implied unsatisfactory, 2 not quite satisfactory, 3 neutral, 4 close to satisfactory and 5 satisfactory, as shown in Figure 3.

\author{
User $>>$ what is gout \\ MedBot>>gout is Hereditary metabolic disorder characterized by recurrent \\ acute arthritis, hyperuricemia and deposition of sodium urate in and around \\ the joints, sometimes with formation of uric acid calculi.
}

$\square$ Satisfactory-5 $\square$ Close to satisfactory-4 $\square$ Neutral-3 $\square$ Not quite satisfactory-2 $\square$ Unsatisfactory -

Figure 3: Sample query and response for expert rating

The average scores of ratings by the three medical experts are given in Figure 4, where Q1 signifies the query category 1: what is, Q2 signifies query category 2: what is the type of, Q3 signifies query category 3: what are the causes of, and Q4 signifies query category 4 : what are the symptoms of. The experts agreed among themselves with a Pearson Correlation Coefficient of 0.54 and $p<0.05$.

Figure 5 shows the number of queries in each category that received an average rating in the various ranges. 


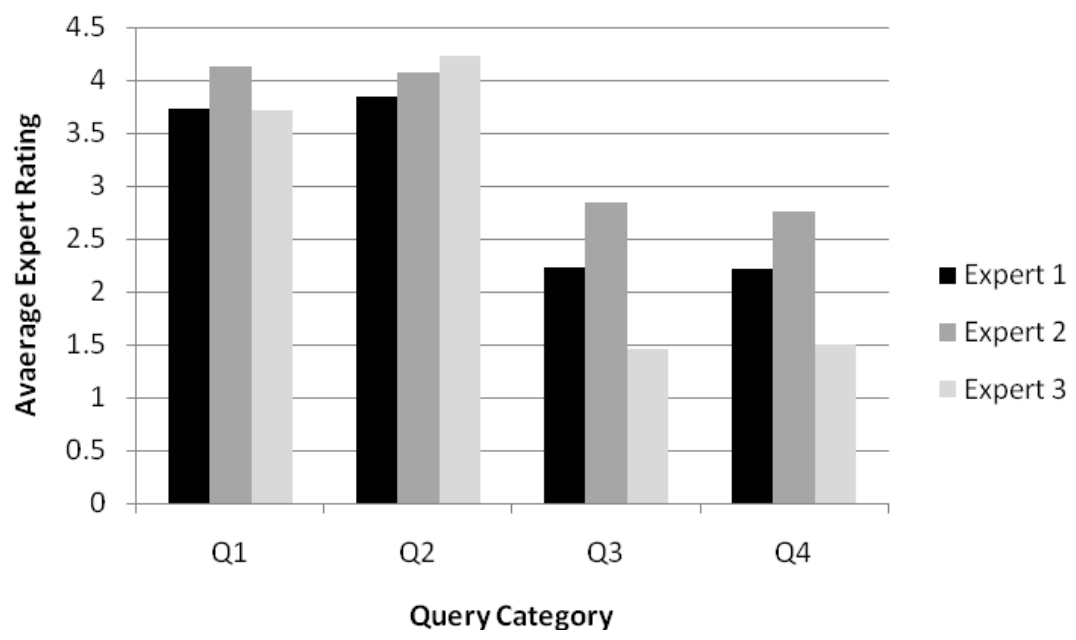

Figure 4: Average Experts Rating of Queries

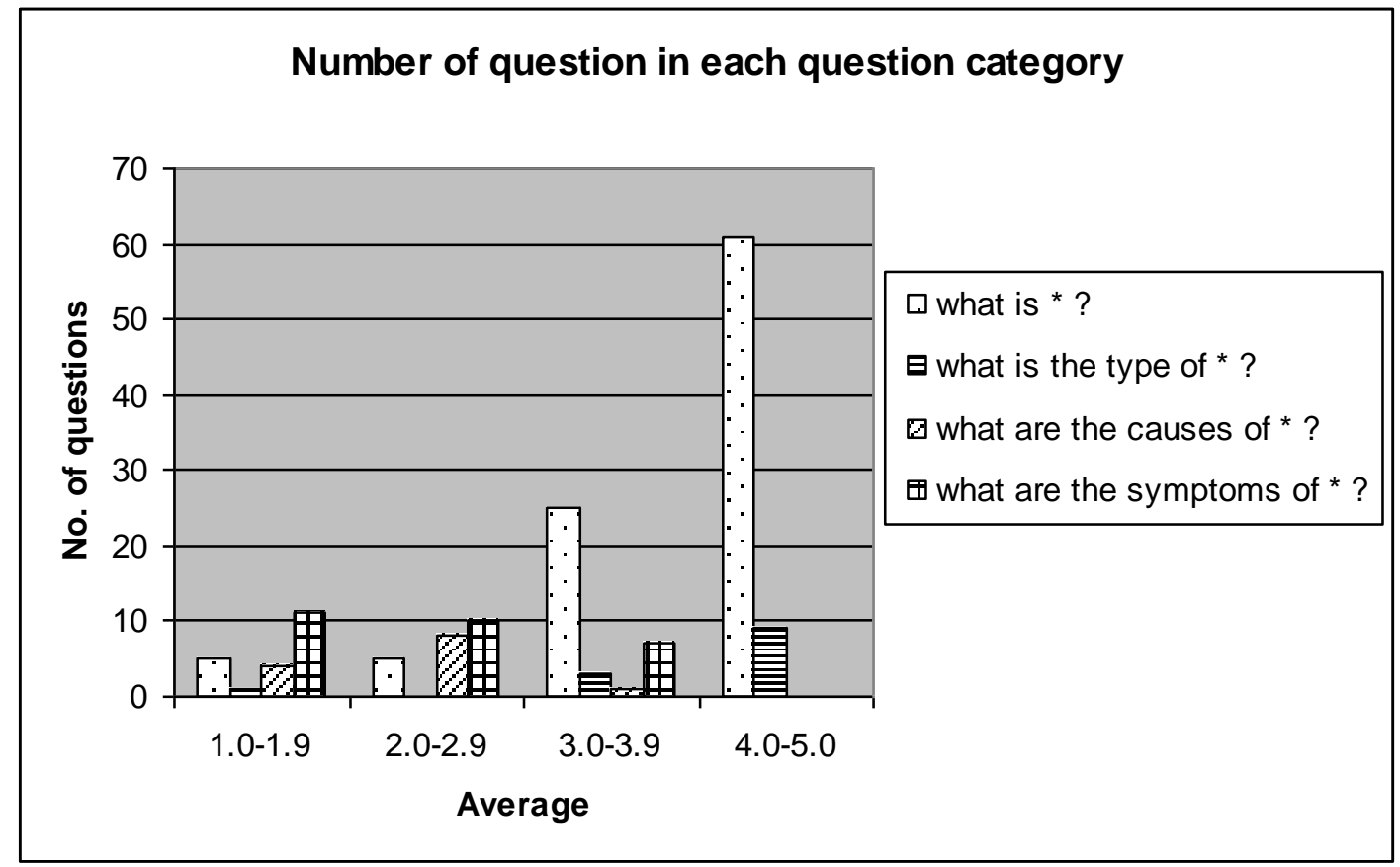

Figure 5: Number of Questions in Rating Ranges

\section{DISCUSSION}

The UMLS [9] is a widely available medical knowledge source and is essentially a collation of various medical ontologies and terminologies (MeSH, SNOMED-CT, Gene Ontology, etc). UMLS contains about two million medical concepts covering various medical domains and 135 semantic types, where each medical concept is assigned at least one semantic type. For example, the concept heart attack has semantic type disease or syndrome, whereas the concept swelling has semantic type finding.
From Figure 4 it is obvious that responses to what is and what is the type of queries received strong acceptance from the experts. On the other hand, responses to what are the causes of and what are the symptoms of queries received low acceptance. This is because knowledge corresponding to the query categories Q3 and Q4 is not explicitly found in the knowledge source and responses are generated through knowledge extraction.

It must be mentioned here that the query responses included cases where UMLS had knowledge gaps. For example, the response to the query what is anemia minor, did not contain any information because UMLS did not have the textual 
definition for the concept anemia minor. Thus if the sample of query responses were to contain only those for which UMLS contains the required knowledge, the results will be much stronger.

\section{CONCLUSIONS}

This paper describes an easy to build chatbot for medical students by deploying the widely available medical knowledge source UMLS and the open source Chatterbean. MedChatBot could be incorporated in a tutoring system to support natural dialog. Although we have described the implementation in the context of the medical domain, the techniques can easily be applied to other domains too. The interface provides for natural dialog interaction, whereas a knowledge source containing textual definition of the domain concepts can be used to generate relevant responses.

In interpreting the results presented above, it must be noted that the UMLS has not been specially designed for the task of supporting natural dialog in chatbots or, providing responses to student queries. A domain knowledge source that is more suited to support such queries is likely to yield better results. A broad knowledge source such as UMLS allows the description of virtually any concept within the broad medical domain. The lack of causal relationships between concepts in UMLS limits the system ability to generate accurate responses to queries such as what are the causes and what are the symptoms. Future editions of UMLS that feature such support can lead to highly accurate responses for such queries.

We intend to incorporate Medchatbot in the existing METEOR [11] tutoring system for medical PBL, which will greatly facilitate natural dialog between the student users and the tutoring system. We also intend to evaluate the student learning outcomes as a result of the natural dialog facility through the chatbot, and compare the results with and without the chatbot.

\section{ACKNOWLEDGMENTS}

We thank the faculty members and students at Isra University, who helped in the user survey and expert ratings of query responses.

\section{REFERENCES}

[1] Weizenbaum, J. (1966). ELIZA - A Computer Program For The Study of Natural Language Communications Between Man and Machine. Communications of the ACM, 1966. 9(1): p. 36-45
[2] Knill O., Carlsson J., Chi A. and Lezama M. (2004). An artifical intelligence experiment in college math education. Retrieved February 3, 2009 from the World Wide Web:

http://abel.math.harvard.edu/ knill/preprints/sofia.pdf

[3] Jia, J. (2004). CSIEC (Computer Simulator in Educational Communication): An Intelligent Web-Based Teaching System for Foreign Language Learning. In EDMEDIA (World Conference on Educational Multimedia, Hypermedia \& Telecommunications). 2004: Lugano, Switzerland.

[4] Kerly, A., Hall, P. \& Bull, S. (2006). Bringing Chatbots into Education: Towards Natural Language Negotiation of Open Learner Models. In R. Ellis, T. Allen \& A. Tuson (eds), Applications and Innovations in Intelligent Systems XIV - Proceedings of AI-2006, 26th SGAI International Conference on Innovative Techniques and Applications of Artificial Intelligence, Springer.

[5] Webber, G. M. (2005). Data representation and algorithms for biomedical informatics applications. $\mathrm{PhD}$ thesis, Harvard University.

[6] Shawar, B., A., and Atwell, E. (2007) Chatbots: are they really useful? Journal of Computational Linguistics and Language Technology, Vol. 22, No. 1, pp.29-49.

[7] Wallace, R. S. (2010). Alicebot. Retrieved June 2, 2010 from the World Wide Web: http://www.alicebot.org/aimlbots.html

[8] Filho, H. P. (2009). ChatterBean: Flexible Alicebot. Retrieved February 3, 2009 from the World Wide Web: http://www.geocities.com/phelio/chatterbean/

[9] U.S. National Library of Medicine. (2012). Unified Medical Language System (UMLS). Retrieved March 18, 2012 from the World Wide Web: http://www.nlm.nih.gov/research/umls/

[10] Liu, Z., \& Chu, W. (2007). Knowledge-based query expansion to support scenario-specific retrieval of medical free text. Information Retrieval, 10(2), April 2007.

[11] Kazi, H., Haddawy, P., Suebnukarn, S. Employing UMLS for Generating Hints in a Tutoring System for Medical Problem-Based Learning. Journal of Biomedical Informatics (2012), 45 (3). 